\title{
A 3D Multi-objective Optimization Planning Algorithm for Wireless Sensor Networks
}

\author{
Danping He, Jorge Portilla, Teresa Riesgo
}

\begin{abstract}
The complexity of planning a wireless sensor network is dependent on the aspects of optimization and on the application requirements. Even though Murphy's Law is applied everywhere in reality, a good planning algorithm will assist the designers to be aware of the short plates of their design and to improve them before the problems being exposed at the real deployment. A 3D multi-objective planning algorithm is proposed in this paper to provide solutions on the locations of nodes and their properties. It employs a developed ray-tracing scheme for sensing signal and radio propagation modelling. Therefore it is sensitive to the obstacles and makes the models of sensing coverage and link quality more practical compared with other heuristics that use ideal unit-disk models. The proposed algorithm aims at reaching an overall optimization on hardware cost, coverage, link quality and lifetime. Thus each of those metrics are modelled and normalized to compose a desirability function. Evolutionary algorithm is designed to efficiently tackle this NP-hard multi-objective optimization problem. The proposed algorithm is applicable for both indoor and outdoor 3D scenarios. Different parameters that affect the performance are analyzed through extensive experiments; two state-of-the-art algorithms are rebuilt and tested with the same configuration as that of the proposed algorithm. The results indicate that the proposed algorithm converges efficiently within 600 iterations and performs better than the compared heuristics.
\end{abstract}

\section{INTRODUCTION}

The major challenge in designing wireless sensor networks (WSNs) is the support of various sensing requirements while coping with the computation, energy, communication and cost constraints. Careful node placement can be a very effective optimization means for achieving the desired design goals. However, optimal node placement is a very challenging problem that has been proven to be NP-Hard for most of the formulations of sensor deployment [1], [2], [3].

The heuristic (LowCost) proposed in [4] is an indoor 3D WSN deploy heuristic. The approach first calculates the coverage to deployment cost ratio for all the candidate points in the deployable area, and sensor nodes are put iteratively to the point with maximum ratio, thus the whole region is covered with the minimum cost at the first step. Then connectivity of the network is checked and extra sensor nodes are added between the unconnected node and its closest connected node, or by moving the unconnected node towards the closest connected node without influencing on the sensing coverage. Theoretically, this approach on one hand manages to cover the area with "minimum cost", on the other hand it is not able to effectively tackle the problem between connectivity with minimum cost. It ignores the impact of network lifetime, moreover, the communication links are established only between LOS nodes which is unpractical in real environment. The MOGA algorithm [5] employs multi-objective genetic algorithm to evolve the decision which is proved to be efficient in solving NP-hard problem. It focuses on maximizing the sensing coverage and prolonging the lifetime with a predetermined number of nodes.

The previous two methods are developed for homogeneous WSNs. However, in many prototypical systems available today, sensor networks consist of a variety of different devices. Nodes may differ in the type and number of attached sensors [6], [7]; some computationally more powerful compute nodes may collect, process, and route sensory data from many more limited sensing nodes [8]. There are several works focusing on planning heterogeneous network, such as in [9], constrained versions of the relay node placement problem is studied and connected placement and survivable problem are tackled with different methods. The work in [10] proposes MultipleObjective Metric (MOM) for base station placement to fairly increase various properties of wireless sensor networks.

Some works are developed to tackle the modelling of sensing signal and radio signal to make the deployment algorithm more practical and accurate. The authors in [11] develop a probabilistic sensing model for sensors with line-ofsight-based coverage (e.g., cameras). In [12], the works aims to achieve k-coverage while minimizing the number of sensor nodes. The coverage problem of wireless sensor networks for the rolling terrains is studied in[13] to derive the general expression of the expected coverage ratio for regular terrains and irregular terrains.

To enhance the WSN lifetime, [14] proposes a deployment strategy with a non-uniform deployment method. To make the deployed network resilient to faults, in [15] the authors present an approach to repair the network with redundant backup nodes and guarantee a specified degree of fault tolerance.

A planning algorithm for 3D space is proposed in this work. It is composed of two steps: the fist step generates a connected network by considering maximum coverage to cost ratio, which provides a good starting point for the next step; Afterwards, a multi-objective evolutionary algorithm is designed to efficiently solve the overall optimization problem on cost, coverage, connectivity, lifetime and link quality. This work integrates a developed 3D ray-tracing method to accurately model the sensing signal and radio signal propagation, based on which, all the important metrics are formulated 
TABLE I. SUMMARY OF IMPORTANT SYMBOLS

\begin{tabular}{|l|l|}
\hline$S_{i}$ & sensor with $I D=i$ \\
\hline$N_{i}$ & node with $I D=i$ and $S \in N$ \\
\hline$A_{s}$ & monitoring area \\
\hline$\Phi_{S_{i}}$ & a set of points that are the covered points of $S_{i}$ \\
\hline$C$ & coverage \\
\hline$L$ & lifetime \\
\hline$A L Q$ & average link quality \\
\hline$D_{C, \text { cost }, L, A L Q}$ & desirability formulation of $C$, cost, $L$ or $A L Q$ \\
\hline
\end{tabular}

practically and appropriately. A desirability function is developed by accumulating all the metrics with different weights. The weights of those metrics can be varied according to the designer's goal. The algorithm converges efficiently thanks to the good initial population in the first step and also because of the effective constraints in the evolution procedure. The algorithm is able to provide multiple solutions that satisfy the desired goal, which offers users the opportunity to choose the most suitable one.

In the remainder of the paper, problems are state out and important metrics are formulated in section 2, the proposed planning algorithm is introduced in section 3 , extensive experiments are realized and results are studied in section 4, and conclusions are drawn in section 5.

\section{FORMULATION OF METRICS}

\section{A. Preliminaries}

We summarize the important symbols that are used in this section in Table I. This algorithm employs a ray-tracing method developed in [16] to trace the propagation paths of signals. Multi-path phenomena occurs in the real radio signal transmission. When direct signal encounters obstacles, reflection and diffraction happen as shown in in Fig. 1. Thus a received signal strength (RSS) at the receiver (RX) is computed by summing the arrived waves vectors. The model of sensing signal which only considers direct path, is simpler than the model of radio propagation.

Definition 1. Covered point: A point $m$ is said to be covered by $S_{i}$, if and only if it is within the sensing range of $S_{i}$ and is not obstructed by any obstacle. $\Phi_{S_{i}}$ represents a set of points that are the covered point of $S_{i}$.

$$
\Phi_{S_{i}}=\left\{m \mid m \in \operatorname{sphere}\left(S_{i}, r_{S_{i}}\right) \wedge m \notin O\left(\overrightarrow{m S_{i}}\right)\right\}
$$

where sphere $\left(S_{i}, r_{S_{i}}\right)$ means the sphere with radius $r_{S_{i}}$ centered at node $S_{i} . O\left(\overrightarrow{m S}_{i}\right)$ indicates whether the sensing path from $m$ to $S_{i}$ is obstructed.

Data are collected and forwarded to BS by using Dijkstra's algorithm to search the path with least distance. Thus the searched paths is applicable to distance vector based routing protocols such as AODV and DSR routing protocols. The distance metric in this work is modelled as the path loss of radio signals which not only reflects the physical distance between transmitter and receiver but also indicates the impact of environment on the link.

\section{B. Cost}

At the pre-deployment phase, WSN designers should take into consideration the available budget. One of the design goal,

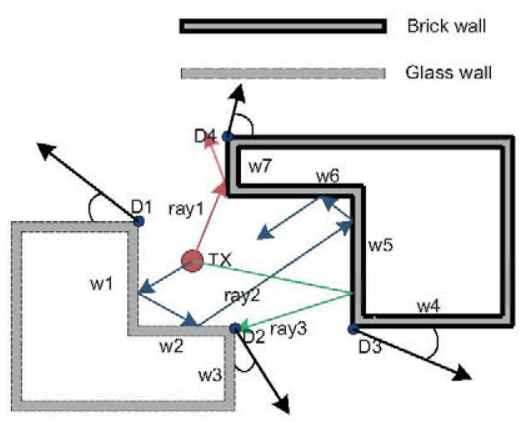

Fig. 1. An example of ray-tracing scheme for signal propagation

from economic point of view, is to reduce the cost while reaching a satisfied performance according to the specification of the application. Many companies and research organizations arise in the recent decade to design and manufacture sensor nodes, which provide various choices to the WSN designers. Nonetheless the deployment cost in different area might vary, there often exist some regions not accessible or is not suitable to deploy nodes. As a result, Cost is categorized into hardware cost and deployment cost.

1) Hardware cost: The newer generations MICAz and Telos support IEEE 802.15.4 and represent a very good solution for generic sensing nodes and are widely implemented. Their unit cost is relatively high. The iMote [17] contains a powerful CPU complemented by a large main memory and non-volatile storage area; on the radio side, Bluetooth has been chosen, thus the price is about \$299. Waspmote [18] works with different communication protocols and frequencies. The flexibility makes the price ranges between $\$ 100 \sim \$ 300$. Some of the manufacturers also produce other types of nodes to cater with the various applications of WSN. WiSGate from Arago Systems and Meshlium produced by Libelium act as gateways.

Due to the heterogeneity property and demanding of various topologies in WSNs, motes with different functions should be considered to obtain an optimum cost solution rather than using a uniform type of full-function sensor mote for the whole sensor network. In this work, motes are classified into three types:

- Sensor Node (SN): equipped with sensors to monitor the surrounding environment. In this work, each sensor node is static and has wireless communication and routing ability.

- Relay Node (RN): has the ability of communication and routing. RN is usually needed to fill the communication hole or to balance the traffic load.

- Base Station (BS): is in charge of aggregating data and is directly connected with the central server. A WSN may have more than one BSs and the location of $\mathrm{BS}$ is predetermined by users.

Since BS is fixed by the WSN designer based on the requirement of application, the cost of $\mathrm{BS}$ is also fixed and is not included in the model of hardware cost. SN contains extra sensor module besides of the communication module, therefore $\mathrm{SN}$ costs more than $\mathrm{RN}$ in our work. The hardware 


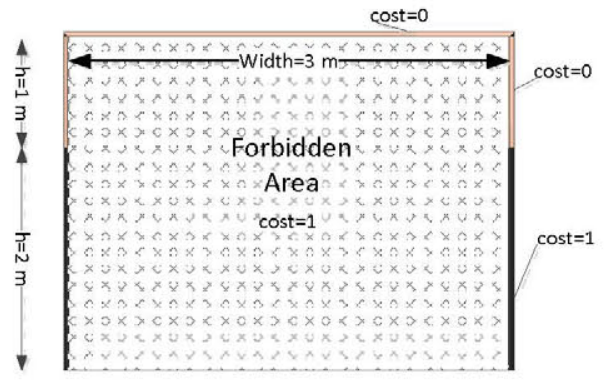

Fig. 2. Deployment cost in vertical view

cost model $C O S T_{h w}$ is expressed by accumulating the price of each node deployed in the target area $A$ :

$$
\operatorname{COST}_{h w}=\sum_{i=1}^{M}\left(P\left(N_{i} . \text { type }\right)\right)
$$

where $P($ type $)$ indicates the price that is dependent on different node types, for instance $P(R N)=100, P(S N)=200$.

The normalization of $C O S T_{h w}$ provides a desirability component of design goal to minimize the cost. By considering the available budget offered by the user as the maximum cost, the $D_{\text {cost }}$ is used to represent the desirability over the hardware cost. The budget can be modelled as a maximum number of sensor nodes allowed in the WSN multiplied by the corresponding price $\operatorname{Max}_{\text {cost }}=N_{\max } \times P(S N)$.

$$
D_{\text {cost }}=\frac{\operatorname{Max}_{\text {cost }}-\operatorname{COST}_{h w}}{\operatorname{Max}_{\text {cost }}}
$$

2) Deployment cost: The lessons learnt by other researcher$s$ indicate that mounting problem occurs during real deployment. From 2004-2005, [netherlands] tried to deploy 150+ sensor nodes to monitor the potato crops for precision agriculture. The original plan was to put nodes below a height of 40 $\mathrm{cm}$, however they discovered at the first attempt that the communications would be obstructed by leaves and flowers as the crops growing. Besides. The nodes will be broken if they were placed within the range of equipment attached to the tractors.

In this work, deployment cost is modeled as boolean value and is assigned by users through GUI interface as shown in Fig. 2, cost $_{d}=1$ if the area is not accessible otherwise $\operatorname{cost}_{d}=0$.

\section{Coverage}

One of the fundamental problems in sensor networks is the coverage problem, which reflects the quality of service that can be provided by a particular sensor network. The coverage concept is defined from several points of view due to a variety of sensors and a wide-range of their applications. With sparse coverage, only parts of the area of interest are covered by the sensor nodes. With dense coverage, the area of interest is completely (or almost completely) covered by sensors. With redundant coverage, multiple sensors cover the same physical location. The actual degree of coverage is mainly determined by the observation accuracy and redundancy required. Coverage may vary across the network. For example, nodes may be deployed more densely at interesting physical locations.
The degree of coverage also influences information processing algorithms. High coverage is a key to robust systems and may be exploited to extend the network lifetime by switching redundant nodes to power-saving sleep modes.

Definition 2. $k$-coverage: A WSN is said to have $k$-coverage if and only if each point in $A_{s}$ is the covered point of $k$ different SNs.

Therefore the desirability of $C$ of $A_{s}$ is expressed as:

$$
D_{C}=\frac{\left|\sum_{i=1}^{M}\left\{m \mid m \in A_{s} \wedge m \in \Phi_{S_{i}}\right\}\right|}{\left|\left\{m \mid m \in A_{s}\right\}\right| \times k}
$$

\section{Connectivity}

Wireless sensor networks are represented by a graph $G=$ $(V, E)$ where $V$ is the set of nodes and $E \subseteq V^{2}$ is the set of edges: $\left(N_{u}, N_{v}\right) \in E$ means that $N_{u}$ and $N_{v}$ are neighbors. The neighborhood set $N(u)$ of a vertex $N_{u}$ is equal to

$$
N_{v}:\left(N_{u}, N_{v}\right) \in E \vee\left(N_{v}, N_{u}\right) \in E
$$

. As aforementioned, radio propagation are modelled by using accurate ray-tracing scheme, Wireless links are determined by received signal strength thus edges are defined as :

$$
E=\left\{\left(N_{u}, N_{v}\right) \in V^{2} \mid u \neq v \wedge R X(u v) \geq R X_{s}\right\}
$$

with $R X(u v)$ being the received signal strength from $N_{u}$ to $N_{v}$, and $R X_{s}$ the sensitivity of antenna at the receiver.

\section{E. Lifetime ratio}

The network longevity is defined as the time that the first node exhausts its energy. We define $N_{j}$ is a child of $N_{i}$ if $N_{i}$ is the in the path from $N_{j}$ to BS. Thus the more children a node has, the sooner it is going to exhaust its battery due to the heavy traffic load. Ideally, the maximum longevity can be achieved when all the nodes communicate directly with the BS. On the contrary, the worst case would occur when a node is the gateway of all the other nodes. The desirability of network longevity is modeled as follow:

$$
D_{L}=\frac{\left(N_{\max }-1\right)-\max \left(C H_{i}\right)}{N_{\max }-1}
$$

where $C H_{i}$ means number of children of $N_{i}$.

\section{F. Average link quality}

This metric evaluates the average communication quality and is obtained by finding the mean path-loss value of all the direct communications determined by the routing protocol.

$$
A L Q=\frac{\sum_{i=1}^{M} \sum_{N_{j} \in N(i)}|R X(i, j)|}{\sum_{i=1}^{M}|N(i)|}
$$

The worst case of $A L Q$ is when all the nodes communicate at $R X_{s}$, the relationship between the desirability of link quality and ALQ is:

$$
D_{A L Q}=\frac{\left(\left|R X_{s}\right|-A L Q\right)}{\left|R X_{s}\right|}
$$

At the end, the entire desirability that represents the design goal is expressed in (7). It is an accumulation of all the 
above mentioned metrics multiplied by corresponding weights. Where $w_{1} \cdots w_{4}$ are the weights and are assigned according to the design goal.

$$
f(C, \operatorname{cost}, L, A L Q)=w_{1} D_{C}+w_{2} D_{\text {cost }}+w_{3} D_{L}+w_{4} D_{A L Q}
$$

\section{PROPOSED METHODOLOGY}

As discussed above, the modelling of important metrics are based on the accurate signal modelling and practical routing protocol, which provides a good starting point to evaluate the impact of deployment solution towards the performance of WSN. By taking advantage of those theoretical analysis, the challenge becomes: Find the locations of nodes and decide their corresponding properties to maximize the desirability value calculated by (7). This multi-objective optimization problem is proven to be NP-hard, and evolutionary algorithm is a proper way to tackle it. A DNA $D V$ is used to express a deployment solution. The format of $D V$ is represented by (8), $N_{i}$ is the node with $I D=i$ which is the "chromosome" of $D V . M$ also indicates the size of WSN. Each node has properties like location, node type, transmission power $P_{t x}$, RX sensitivity $R X_{s}$ and sensing range $R_{\text {sense. The fitness value }}$ is calculated and compared among the parent and children at each generation. In this work, the fitness function employs the desirability format (7).

$$
D V=\left[N_{1}, N_{2}, N_{3} \cdots N_{M}\right], N_{i}=\left\{\begin{array}{l}
\text { location }: x, y, z \\
\text { type }: B S, S N, R N \\
P_{t x}, R X_{s} \\
R_{\text {sense }}
\end{array}\right\}
$$

Theoretically, GA algorithm is able to provide a solution that has the best desirability if the number of generation is big enough. However, the time consumption due to this timeless iteration will exhaust users' passion. To overcome this problem, there are several ways such as limiting the maximum number of generation, setting a threshold for the desirability, and so on.

In this work, a merit seed for the whole procedure is generated by considering fulfill the coverage and connectivity. At the beginning, the Coverage is computed for each deployable point $m \in A$ and the algorithm selects $m_{i}$ with the maximum coverage area. A sensor node $S_{u}$ is then placed on $m_{i}$. The coverage level of the monitoring points newly covered by $S_{u}$ is updated, and those points with a sufficient coverage level are removed from the set of $A_{s}$. The above steps are repeated until all the monitoring points are sufficiently covered. Afterwards, the algorithm focuses on the connectivity problem. Let $N_{u}$ be the node element of unconnected nodes $U$ under consideration. The algorithm selects a node $N_{c}$ in the connected sensor nodes $C$ that is the closest to $N_{u}$ and computes the new virtual position $m^{\prime}$ of $N_{u}$ by moving it towards $N_{c}$ as long as the set of monitoring points initially covered by $N_{u}$ remains unchanged. If the sensor node $N_{u}$ is still unconnected after changing its position, extra relay nodes are put on the line segment with endpoints $N_{u}$ and $N_{c}$ so that $N_{u}$ and $N_{c}$ are connected. The resulted the initial seed is expected to be better than a randomly generated seed that without guaranteeing the coverage and connectivity.
Afterwards, the genetic algorithm starts to adjust the entire topology to obtain higher fitness value. At each iteration, the mutations occur with different probabilities on the $3 \mathrm{D}$ location and on the type property. If the mutation occurs on the location, the movement is limited within the region of a maximum 3D distance $d_{\max }$ from the original point. The type can be selected between $\mathrm{SN}$ and $\mathrm{RN}$ if mutation of node type happens. Then routing protocol is applied and routs are searched for each node to reach the BS. A SN is changed to a RN when it does not cover any point $m$ in the $A_{s}$. And a $\mathrm{RN}$ is deleted when it is the leaf node of a link to the BS. $q$ children are generated at each generation based on the above rules and are evaluated by (7), and the best child is selected as a parent for the next generation. This procedure repeats until the maximum generation is reached. Different results will be obtained by running the same algorithm with same configurations. However, this feature gives users a flexibility to make their decision.

\section{EXPERIMENTS AND ANALYSIS OF THE RESULTS}

The planning algorithm is implemented in $\mathrm{C}++$. In this section, experiments are realized to study the performance by varying different parameters. The proposed algorithm is compared with two 3D deployment heuristics in indoor environment.

\section{A. Effects of different parameters}

The tests are realized in an indoor office environment that contains rooms and furniture, and the size of target space is $57 m \times 16 m \times 3 \mathrm{~m}$, Several parameters are set to be uniform for antennas, including the $R X_{s}=-90 \mathrm{~dB}$, transmission power $P_{t x}=0 d B$, the resolution is set to be $1 \mathrm{~m}$. Fig. 6 shows the environment and user configurations, the monitoring regions are those points within the rectangles.

1) Maximum Number of Generation: The fitness value progresses as number of generation increases. In this test, the number of children generated at each iteration is $q=6$. The Maximum value of generation $\max _{g r}$ increases from 100 to 1000 with a step of 100 , hence there are 10 different $\max _{g r} \mathrm{~s}$. The algorithm runs 30 times for each $\max _{g r}$, the resulted 30 best $f s$ are grouped for each $\max _{g r}$ and shown by box plots (Fig. 3). As can be seen, the mean value of $f$ increases as max $_{g r}$ increases and the performance becomes more stable when $M a x_{\text {generation }} \geq 600$.

Fig. 4 shows how each metric varies with $\max _{g r}$. The $D_{C}=1$ for all the time as it is set to be the most important metric among the others, while on the contrary and $D_{A Q L}$ have the least impact on $f$.

The time spent on running the algorithm increases with $\max _{g r}$ and it is impractical to run with a large generation to obtain an ideal result. We observe that when $\max _{g r} \geq 600$, all the desirability values increases much slower and are almost stable. Hence, the value can be used in the planning algorithm for the following tests.

2) The weights $w_{i}$ : The weights describe the importance of each metric in the WSN. Sometimes, cost should be emphasized while sometimes lifetime is more important than the cost. We vary the $w_{i}$ with the purpose to study how 


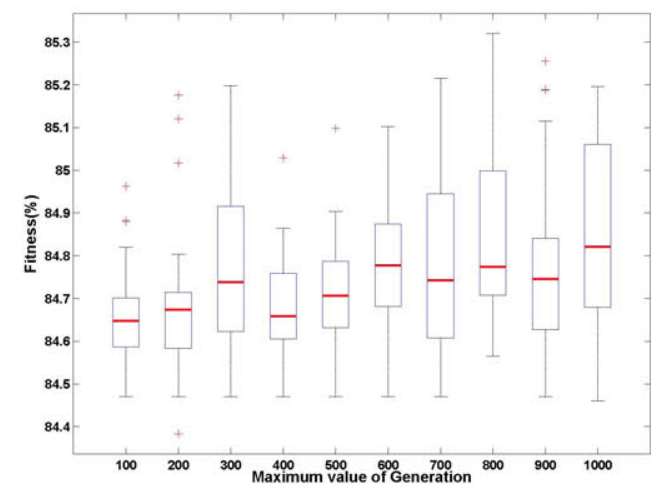

Fig. 3. $f$ vs $\max _{g r}$

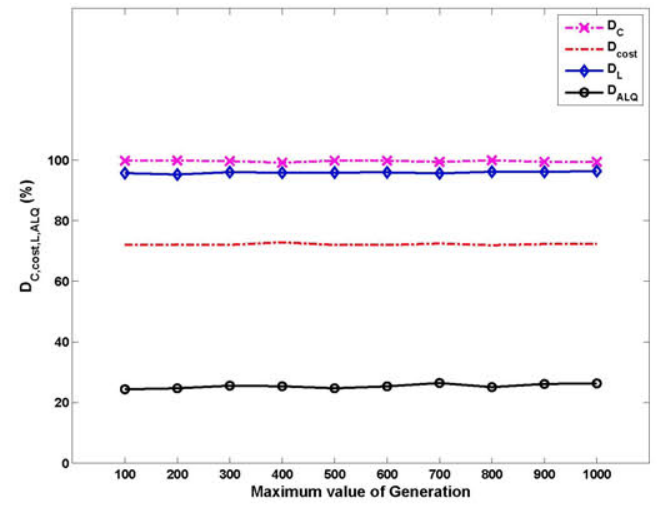

Fig. 4. Different metrics vs $\max _{g r}$

TABLE II. VALUES OF WEIGHTS

\begin{tabular}{|c|c|c|c|c|}
\hline Case & $w_{1}$ & $w_{2}$ & $w_{3}$ & $w_{4}$ \\
\hline I & $50 \%$ & $25 \%$ & $15 \%$ & $10 \%$ \\
II & $70 \%$ & $20 \%$ & $5 \%$ & $5 \%$ \\
III & $20 \%$ & $50 \%$ & $20 \%$ & $10 \%$ \\
IV & $25 \%$ & $25 \%$ & $25 \%$ & $25 \%$ \\
\hline
\end{tabular}

they affect the metrics. Table II are the values of weights of four different cases. Fig. 5(a) shows the progressing of $f$ for different cases, case II has the best fitness value while case IV performs the worst by averaging the weights. In case III and $\mathrm{IV}$, the coverage is not guaranteed due to the small value of $w_{1}$ (see Fig. 5 (b)). Fig. 5(d) shows that no matter how the value of $w_{3}$ varies the $D_{L}$ of the four cases overlap together and are not distinguishable. Therefore $D_{L}$ is constraint significantly by other metrics. In terms of communication quality (Fig. 5(e)), case IV outperforms the others with highest cost and lowest coverage, thus the extra nodes are added for enhance the link quality rather than for the coverage.

\section{B. Comparison with other algorithms}

Both LowCost [4] and MOGA[5] are rebuilt in this work. Notice that their sensing and radio signals models are not practical, we modify them by using our models for fairly comparison without limiting their performance on planning.
TABLE III. THE RESULT MATRIX OF THE ALGORITHMS

\begin{tabular}{|c|c|c|c|c|c|}
\hline Algorithm & $D_{C}$ & $D_{\text {cost }}$ & $D_{L}$ & $D_{A L Q}$ & $f$ \\
\hline Proposed & $100 \%$ & $66 \%$ & $98 \%$ & $22 \%$ & $83.4 \%$ \\
LowCost & $100 \%$ & $70 \%$ & $86 \%$ & $22 \%$ & $82.6 \%$ \\
MOGA & $100 \%$ & $70 \%$ & $88 \%$ & $19 \%$ & $82.6 \%$ \\
\hline
\end{tabular}

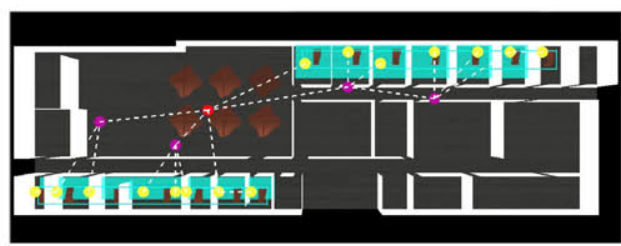

Fig. 6. Solution generated by proposed algorithm

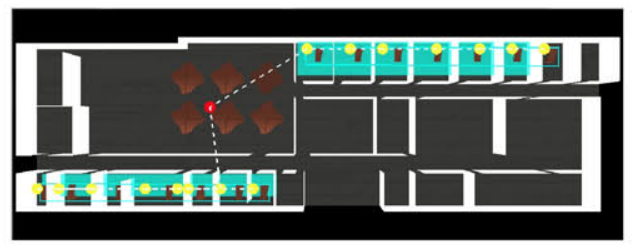

Fig. 7. Solution generated by LowCost

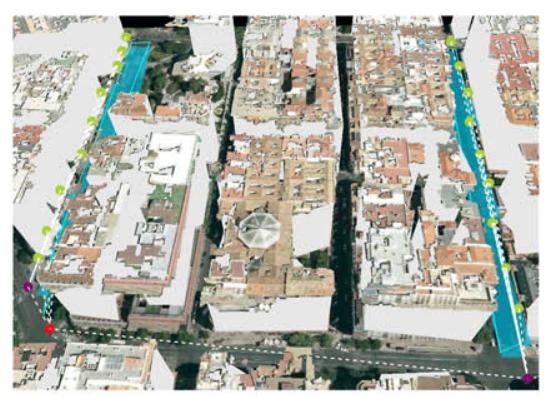

Fig. 8. Outdoor solution generated by proposed algorithm

Besides the number of nodes are fixed in MOGA and obviously the value depends a lot on the specification of WSN application. To overcome this disadvantage, we determine the proper value by using the number obtained by LowCost. Fig. 6 and Fig. 7 are the results of the proposed algorithm and LowCost respectively, due to the limits on pages, the result of MOGA is not presented. Table III concludes the results of the three methods with the same configuration. The proposed algorithm performs the best which places 4 relay nodes to balance the traffic and improve the communication quality. The LowCost only considers coverage and cost, thus 2 of the sensor nodes, which act as gateways, are likely to exhaust their energy very soon. MOGA focuses on the coverage and lifetime with a fixed number of node. Although the lifetime is slightly better than LowCost while the link quality is worse. Although MOGA takes advantage of LowCost on determining the size of network, it does not performs better than LowCost. Fig. 8 is an example of the planning for outdoor environment by the proposed algorithm.

\section{CONCLUSION}

A planning algorithm is proposed in this paper, By taking the advantage of proposed ray-tracing scheme for both radio 


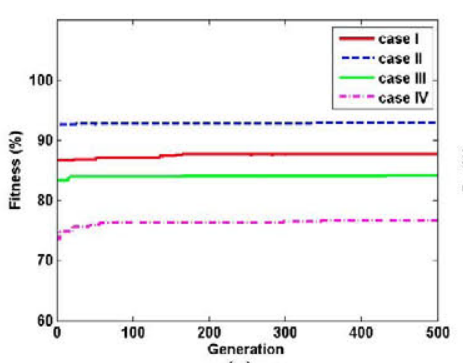

(a)

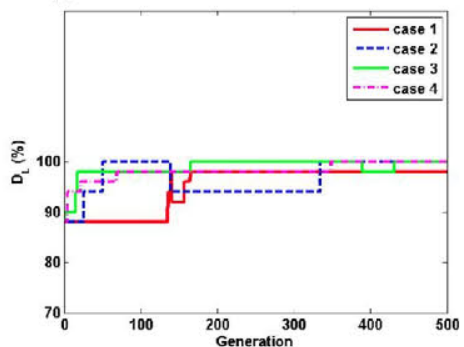

(d)

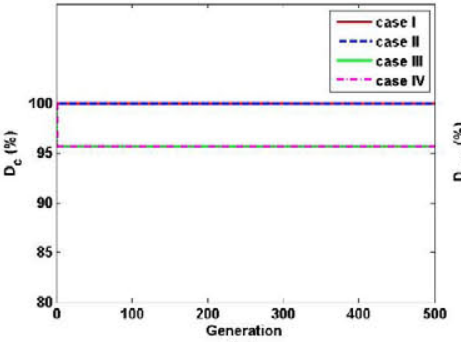

(b)

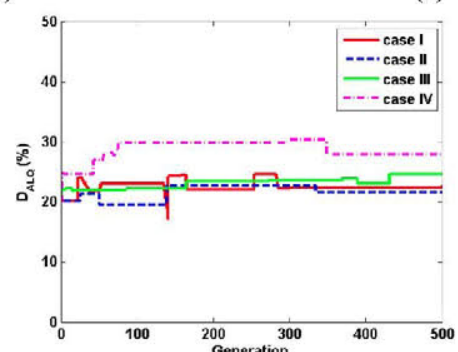

(e)

Fig. 5. (a) $D$ VS Generation with different weights; (b) $D_{C}$ VS Generation with different weights; (c) $D_{\text {cost }}$ VS Generation with different weights; (d) $D_{L}$ VS Generation with different weights; (e) $D_{A L Q}$ VS Generation with different weights

and sensing siganl propagation, the modeling on coverage and connectivity is accurate. Moreover, we also consider the important impact of lifetime and link quality on the WSN, the optimization is more complete compared with other works. This algorithm is suitable for both outdoor and indoor environment with the ability to consider deployable area and forbidden area. In the future, scalability and probabilistic model of sensing signal will be studied to further improve the algorithm.

\section{ACKNOWLEDGMENT}

The authors would like to acknowledge the support of WSN DPCM project, ARTEMIS JU and Spanish Ministry of Industry and commerce under grant ART-010000-2011-1.

\section{REFERENCES}

[1] A. Efrat, S. Har-peled, and J. S. B. Mitchell, "Approximation algorithms for two optimal location problems in sensor networks," 2004.

[2] X. Cheng, D.-Z. Du, L. Wang, and B. Xu, "Relay sensor placement in wireless sensor networks," Wirel. Netw., vol. 14, no. 3, pp. 347-355, Jun. 2008. [Online]. Available: http://dx.doi.org/10.1007/s11276-0060724-8

[3] S. Poduri, S. Pattem, B. Krishnamachari, and G. S. Sukhatme, "Sensor network configuration and the curse of dimensionality," 2006.

[4] M. T. Kouakou, S. Yamamoto, K. Yasumoto, and M. Ito, "Cost-efficient deployment for full-coverage and connectivity in indoor 3d wsns," in Proc. IPSJ Dicomo 2010, 2010.

[5] D. Jourdan and O. de Weck, "Layout optimization for a wireless sensor network using a multi-objective genetic algorithm," in Proc. Vehicular Technology Conference, 2004. VTC 2004-Spring. 2004 IEEE 59th, vol. 5, 2004, pp. 2466-2470 Vol.5.

[6] Smartsantander. http://www.smartsantander.eu/. [Online]. Available: http://www.smartsantander.eu/

[7] Citysense. https://www.sensenetworks.com/products/macrosensetechnology-platform/citysense/.

[8] M. Ahlberg, V. Vlassov, and T. Yasui, "Router placement in wireless sensor networks," in Proc. MASS, 2006, pp. 538-541.
[9] S. Misra, S. D. Hong, G. Xue, and J. Tang, "Constrained relay node placement in wireless sensor networks: Formulation and approximations," Networking, IEEE/ACM Transactions on, vol. 18, no. 2, pp. 434 447, 2010.

[10] S. Kim, J.-G. Ko, J. Yoon, and H. Lee, "Multiple-objective metric for placing multiple base stations in wireless sensor networks," in Proc. Wireless Pervasive Computing, 2007. ISWPC '07. 2nd International Symposium on, 2007.

[11] V. Akbarzadeh, C. Gagne, M. Parizeau, M. Argany, and M. Mostafavi, "Probabilistic sensing model for sensor placement optimization based on line-of-sight coverage," Instrumentation and Measurement, IEEE Transactions on, vol. 62, no. 2, pp. 293-303, 2013.

[12] S. Xiong, L. Yu, H. Shen, C. Wang, and W. Lu, "Efficient algorithms for sensor deployment and routing in sensor networks for networkstructured environment monitoring," in Proc. INFOCOM, 2012 Proceedings IEEE, 2012, pp. 1008-1016.

[13] L. Liu and H. Ma, "On coverage of wireless sensor networks for rolling terrains," Parallel and Distributed Systems, IEEE Transactions on, vol. 23, no. 1, pp. 118-125, 2012.

[14] S. Qian, P. Guo, and T. Jiang, "A novel lifetime-enhanced deployment strategy for chain-type wireless sensor networks," in Proc. Communications (ICC), 2012 IEEE International Conference on, 2012, pp. 513517.

[15] M. Bhuiyan, J. Cao, and G. Wang, "Deploying wireless sensor networks with fault tolerance for structural health monitoring," in Proc. Distributed Computing in Sensor Systems (DCOSS), 2012 IEEE 8th International Conference on, 2012, pp. 194-202.

[16] D. He, G. Liang, J. Portilla, and T. Riesgo, "A novel method for radio propagation simulation based on automatic $3 \mathrm{~d}$ environment reconstruction," Radioengineering, vol. 21,4, pp. 985-992, December 2012.

[17] R. M. Kling, "Intel mote: An enhanced sensor network node," in Proc. of the International Workshop on Advanced. Sensors, Structural Health Monitoring, and Smart Structures, Raiosha, Japan, Nov. 2003, pp. 1-6. [Online]. Available: http://www.mita.sd.keio.ac.jp/news/workshop/proceedings.htm

[18] Waspmote - wireless sensor networks 802.15.4 zigbee mote. http://www.libelium.com/products/waspmote. 